\title{
BMJ Open Effectiveness of evidence-based treatments of fetal alcohol spectrum disorders in children and adolescents: a systematic review protocol
}

\author{
Deepa Singal, ${ }^{1}$ Chantalle Menard, ${ }^{2}$ Christine J Neilson, ${ }^{3}$ Marni Brownell, ${ }^{1}$ \\ Ana Hanlon-Dearman, ${ }^{4}$ Albert Chudley, ${ }^{4}$ Ryan Zarychanski, ${ }^{5,6}$ Ahmed Abou-Setta ${ }^{7}$
}

To cite: Singal D, Menard C, Neilson CJ, et al. Effectiveness of evidence-based treatments of fetal alcohol spectrum disorders in children and adolescents: a systematic review protocol. BMJ Open 2018;8:e013775. doi:10.1136/ bmjopen-2016-013775

- Prepublication history for this paper is available online. To view these files, please visit the journal online (http://dx.doi org/10.1136/bmjopen-2017013775).

Received 5 August 2016 Revised 3 December 2017 Accepted 6 December 2017

Check for updates

For numbered affiliations see end of article.

Correspondence to

Dr Deepa Singal;

DeepaKapoorSingal@gmail.com

\section{ABSTRACT}

Introduction The aim of this paper is to provide a protocol for a systematic review assessing the effectiveness of evidence from randomised controlled trials comparing fetal alcohol spectrum disorders pharmacological and non-pharmacological interventions with placebo/dummy interventions or usual standards of care in children and adolescents ( $<18$ years old).

Methods and analysis The following electronic databases will be searched: Medline (Ovid), Cumulative Index of Nursing and Allied Health Plus with Full text (EBSCO), Cochrane Central Register of Controlled Trials (Cochrane Library-Wiley), PsycINFO (ProQuest) and Proquest DissertationsandTheses will be searched from inception to March 2017 for relevant citations of published trials using individualised search strategies prepared for database. We will also search the reference lists of relevant articles and conference proceedings. Two reviewers will independently assess each study against predetermined inclusion/exclusion criteria and extract data including population characteristics, types and duration of interventions and outcomes from included trials. Internal validity will be assessed using the Cochrane Risk of Bias Tool. Primary outcome measures will be improvements in symptoms, including: hyperactivity, impulsivity and attention as measured by standard rating scales. Secondary outcome measures will include improvements in physical and mental health domains, as well as cognitive, behavioural, social and educational skills as measured by rating scales, standardised psychometric tests of $I Q$ and memory, grade repetition, literacy tests and diagnosis of mental health disorder.

Ethics and dissemination Ethical approval will not be obtained since it is not required for systematic reviews as there are no concerns regarding patient privacy. The results of this review will be disseminated through publication in a peer-review journal and presented at relevant conferences.

PROSPERO registration number CRD42013005996.

\section{INTRODUCTION}

Fetal alcohol spectrum disorder (FASD) is diagnostic term comprising a broad range of symptoms and disabilities associated
Strengths and limitations of this study

- This systematic review will include a thorough search of the literature including searching multiple bibliographic databases, hand-searching relevant conference proceedings, included studies and previous reviews, forward searching and searching clinical trials registry for ongoing trials. In addition, authors of ongoing studies will be contacted to obtain unpublished results.

- This review will follow established methodological guidelines in the conduct and reporting of this review, that is, the Methodological Expectations for Cochrane Interventional Reviews for conduct and reporting of reviews.

- Study selection, data extraction and assessment of trial internal validity will be performed independently by two researchers. This will ensure the absence of personal bias in the inclusion and assessment of studies.

- Further limitations may also arise from the fact that there is high heterogeneity from the methodology used to evaluate fetal alcohol spectrum disorder treatments.

with prenatal alcohol exposure. ${ }^{12}$ Diagnosis requires a neurodevelopmental assessment conducted by a multidisciplinary team and includes a social and medical history, along with complete physical examination. ${ }^{2}$ Patients with a diagnosis of FASD must have the confirmation of prenatal alcohol exposure and may have sentinel facial features and/or evidence of impairment in neurodevelopmental domains. ${ }^{2}$ More information regarding diagnosing FASD is available in the updated Canadian FASD guidelines. ${ }^{2}$

FASD has been estimated at 5 in 1000 people in Canada and 15 in 1000 people in the $\mathrm{USA}^{3}$ and has been recognised as the leading preventable cause of intellectual disability in North America, ${ }^{4}$ indicating FASD is a significant public health issue. ${ }^{5}$ Children 
diagnosed with FASD may experience a myriad of primary and secondary conditions. Primary disabilities are related to central nervous system dysfunction and vary according to the degree of neurodevelopmental damage that has occurred. ${ }^{6}$ These disabilities can include intellectual disability, low IQ, impaired executive functioning, memory process and attention, hyperactivity and impulsivity, speech and language difficulties and attention-deficit/ hyperactivity disorder (ADHD). ${ }^{16-8}$ Secondary conditions are associated difficulties that patients with FASD may develop throughout their lifespan, including: mental health disorders such as conduct disorder, depressive disorder and oppositional defiant disorder, difficulties in school including withdrawal and suspension, trouble with the justice system, deviant sexual behaviour, substance abuse issues and employment challenges. ${ }^{9-11}$

Due to these complex health effects and range of expression and disability related to prenatal alcohol exposure, FASD is a difficult condition to diagnose and often goes under-reported and untreated. ${ }^{112}$ Children are often not diagnosed in infancy but may be diagnosed later at school age when symptoms begin to show. ${ }^{6}{ }^{13}$ Furthermore, the range and heterogeneity in symptom severity and presentation between patients makes the clinical management of FASD a tremendous challenge.

Currently, there is no 'gold standard' of treatment of FASD; treatment is multifaceted and multidisciplinary, with the goal of improving symptoms on a case-per-case basis. Treatments can be categorised into pharmacological and non-pharmacological interventions. Pharmacological interventions are usually required to treat comorbid conditions such as depression and ADHD and include stimulant medications, selective serotonin reuptake inhibitors and tricyclic antidepressants. ${ }^{14-16}$ Non-pharmacological interventions include educational and learning strategies, cognitive-behavioural therapy, speech, occupational and physiotherapies and psychosocial interventions.

Several narrative reviews have been published that summarise FASD interventions. ${ }^{14-17}$ While these reviews contribute important insight and frameworks to guide future interventions, they were not conducted using standard systemic review guidelines and may therefore contain bias in their knowledge synthesis. There have been two systematic reviews published on FASD interventions. The first review was published by Peadon et $a l .{ }^{15}$ Peadon $e t a l^{15}$ evaluated both pharmacological and non-pharmacological interventions for children with FASD. Since then, several new studies have been added to the literature. A more recent systematic review ${ }^{20}$ did not review pharmacological interventions, which are an integral and necessary part of treating patients with FASD. Pharmacological treatment is especially relevant for clinicians treating children and adolescents with FASD since a substantial proportion of children diagnosed with FASD also have a comorbid diagnosis of ADHD. ${ }^{810112122}$ Studies have shown up to $94 \%$ of children with heavy prenatal alcohol exposure are diagnosed with ADHD, ${ }^{811} 22$ which is characterised by symptoms of hyperactivity, impulsivity and/or inattention. ${ }^{8}$ These patients are frequently prescribed stimulant medications. ${ }^{23-25}$

To the best of our knowledge, there is no current systematic review evaluating the effectiveness and safety of both pharmacological and non-pharmacological interventions for children with FASD.

\section{Objectives}

This paper describes the protocol for a systematic review that will identify, critically appraise and meta-analyse data (if appropriate) from prospective randomised trials comparing FASD interventions with placebo, usual standards of care or no treatment. The review will assess how effective pharmacological and non-pharmacological interventions are in improving cognitive, psychological and behavioural symptoms of children and adolescents with a diagnosis of FASD compared with other therapies, placebo or to no intervention

\section{METHODS AND ANALYSIS}

\section{Inclusion and exclusion criteria for considering studies for}

\section{this review}

Types of studies

All trials meeting the inclusion criteria, including parallel, cross-over and cluster randomised control trials (RCTs) will be included. There will be no language restrictions (see table 1).

\section{Types of participants}

Children ( $\leq 18$ years), both males and females, with an author defined diagnosis of FASD (ie, studies that state they are using a cohort of children with FASD) including but not limited to: fetal alcohol syndrome, partial fetal alcohol syndrome, alcohol-related neurodevelopmental disorder and alcohol-related birth defects (see table 1).

\section{Types of interventions}

All pharmacological or non-pharmacological interventions targeting the improvement of FASD symptoms in children in all types of intervention settings will be included (see table 1):

- Pharmacological interventions: any pharmacological interventions including, but not limited to: stimulants, antidepressants, neuroleptics and antianxiety drugs (eg, methylphenidate, pemoline, atomoxetine, dextroamphetamine);

- Non-pharmacological interventions: any psychological or social interventions including, but not limited to: cognitive control therapy, education and learning strategies, language and literacy therapy, speech, occupational and physiotherapies, early intervention programmes and psychosocial interventions. Trials evaluating the effect of nutritional supplements (such as choline) will also be included. Non-pharmacological interventions will be grouped according to categorisation by type of intervention, that is, behavioural 
Table 1 Study eligibility criteria

\section{Inclusion criteria}

1. Prospective, randomised controlled trial

2. Patients with a diagnosis of fetal alcohol spectrum disorders, including: fetal alcohol syndrome, partial fetal alcohol syndrome, alcohol-related neurodevelopmental disorder, alcohol-related birth defects, fetal alcohol effects

3. Majority ( $>80 \%$ ) of patients under the age of 18 years of age at time of randomisation

4. Pharmacological interventions including but not limited to:

stimulants, antidepressants, neuroleptics, antianxiety drugs

5. Non-pharmacological interventions but not limited to: cognitive control therapy, education and learning strategies, language and literary therapy, speech, occupational and physiotherapies, early intervention programmes and psychosocial interventions

\section{Exclusion criteria}

\section{Studies involving animals}

2. Non- randomised controlled trials(eg, cohort studies with preintervention and postintervention measurements and case-control studies) intervention, educational intervention and social intervention.

\section{Types of comparators}

Any comparator including standard care, no intervention or placebo/sham intervention.

\section{Types of outcome measures}

This review will evaluate all outcomes pertaining to the children's physical and mental health, as well as cognitive, behavioural and social skills which are presented in studies with the objective of investigating FASD interventions. These outcomes may be measured using standardised/ non-standardised and validated/unvalidated measures, for example, by rating scales (eg, Child Behaviour checklist). All outcome measures included in the studies will be reported in this review. Furthermore, studies will not be excluded on the basis of outcomes; if any of the primary or secondary outcomes are reported, the study is eligible for inclusion. Follow-up data will be collected from all reported time periods, that is, outcomes that are measured during, immediately or after the intervention versus later in life. It is important to note that measures used in this field of study tend to be variable and depend on the intervention targets. We have provided examples of measures that may be included in studies, however, we do not intended this list to be exclusive and will include trials with any standardised measures, including Eyberg Child Behaviour Inventory, Child Behaviour Checklist (CBCL), Social Skills Rating System (SSRS).

\section{Primary outcomes}

1. Behaviour and social skills: measured by rating skills (eg, Personal Behaviour Checklist scores, CBCL, SSRS);

2. Cognitive abilities: measured by psychometric tests of IQ and memory (eg, Ballard Addition and Subtraction Tests);

3. Educational skills and attainment: measured by grade repetition, special educational supports and validated scales measuring literacy and mathematical skills (eg, Phonological Awareness and Early Literacy Test).

\section{Secondary outcomes}

1. Diagnosis of ADHD: measured by clinical diagnosis and assessment;

2. Psychiatric comorbidity: measured by rating scales (eg, Child Depression Inventory, Beck Depression Inventory);

3. Hyperactivity: as measured by rating scales (eg, Conner's Parent Rating Scale (CPRS), Conner's Teacher Rating Scale (CTRS));

4. Impulsivity: as measured by rating scales (eg, CPRS, CTRS);

5. Attention: as measured by rating scales (eg, CPRS, CTRS).

\section{Adverse outcomes}

1. Side effects of pharmacological treatments including, but not limited to: cardiovascular effects, seizures, weight changes and anxiety as measured by side effect symptoms checklists (eg, Barkley Side Effects Questionnaire);

2. Side effects of non-pharmacological treatments including, but not limited to: increase in symptoms or development of new symptoms caused by the behavioural/psychological intervention including, increase in psychiatric symptoms and agitation, as well as possibilities of child maltreatment and/or suicidal ideation/attempts.

\section{Search methods for identification of studies}

\section{Electronic searches}

A search strategy will be designed by a health librarian, using the Embase (Ovid) bibliographic database (see table 2 for a sample search strategy). The strategy will incorporate terminology related to FASD and prenatal alcohol exposure, as well as a paediatric search filter adapted for Embase from Boluyt et $a l_{.}{ }^{26}$ A modified version of the Scottish Intercollegiate Guidelines Network filter for RCTs ${ }^{27}$ 


\begin{tabular}{llr}
\hline \multicolumn{2}{l}{ Table 2} & Embase (Ovid) search strategy \\
\hline$\#$ & Searches & Results \\
\hline 1 & 'f?etal alcohol'.ti,ab,kw,hw. & 7590 \\
2 & FASD.ti,ab,kw,hw. & 1882 \\
& $\begin{array}{l}\text { 1 or 2 [Fetal alcohol spectrum } \\
\text { disorder+variants] }\end{array}$ & 7791
\end{tabular}

(alcohol $^{\star}$ and (neonat ${ }^{\star}$ or prenatal ${ }^{*}$

or natal ${ }^{\star}$ or postnatal ${ }^{*}$ or perinatal ${ }^{\star}$

or pregnan* or 'in utero' or f?etus* or

f?etal) and (expos* or affect* or induc $\left.{ }^{\star}\right)$ ).

4 ti,ab,kw,hw. [prenatal alcohol exposure]

14264

((development ${ }^{\star}$ or neurodevelopment*)

adj3 (f?etus or f?etal or disorder* or

brain or cognitiv* or neurocognitiv*

or neurobehav* or behavio? ${ }^{\star}$ or

neurodevelopment*)).ti,ab,kw,hw.

5 [developmental disorders]

179497

((disorder* or defect* or deficit* or impair*

or anomal* or abnormal* or delay*) adj3

(birth or congenital* or brain or cognitiv*

or neurocognitiv* or neurobehav* or

behavio? $r^{\star}$ or emotion* or social ${ }^{\star}$ or

'self regulat*' or 'executive function*'

or neurodevelopment" or 'motor

function"' or 'motor skill" )).ti,ab,kw,hw.

6 (developmental disorders)

488755

3 and (5 or 6) [developmental disorders \&

7 prenatal alcohol exposure]

4704

83 or 7

9962

infant/or child/orexp childhood/or

adolescent/or adolescence/or 'minor

(person)"/or Puberty/orexp pediatrics/

or school/orhigh school/or kindergarten/

or middle school/ornursery school/

or primary school/or (infant* or infancy

or newborn* or baby* or babies or

neonat ${ }^{\star}$ or preterm* or prematur ${ }^{\star}$ or

postmatur $^{\star}$ or child* or schoolchild ${ }^{\star}$ or

school age $^{*}$ or preschool ${ }^{\star}$ or kid or kids

or toddler* or adoles ${ }^{\star}$ or teen* or boy ${ }^{\star}$ or

girl $^{\star}$ or minors or pubert* or pubescen*

or p?ediatric* or pe?diatric* or nursery

school* $^{*}$ or kindergar* or primary school ${ }^{\star}$

or secondary school* or elementary

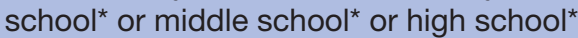

9 or highschool*).ti,ab,kw,hw. [child filter] 4052596

108 and 9 [FASD+Children] 5889

10 not ((exp animal/or nonhuman/) not

11 human/)

4873

12 exp clinical trial/ 1331190

13 Randomized controlled trial/ 482169

14 controlled study/ 5520031

15 multicenter study/ 166062

16 Randomization/ 85018

\begin{tabular}{llr}
17 & Single blind procedure/ & 29898 \\
18 & Double blind procedure/ & 141530 \\
\hline
\end{tabular}

Continued
Table 2 Continued

\begin{tabular}{|c|c|c|}
\hline \# & Searches & Results \\
\hline 19 & Crossover procedure/ & 55426 \\
\hline 20 & Prospective study/ & 402295 \\
\hline 21 & Placebo/ & 333602 \\
\hline 22 & random*.ti,ab. & 1183145 \\
\hline 23 & trial*.ti. & 299817 \\
\hline 24 & (Rct or RCTs).ti,ab. & 45861 \\
\hline 25 & Random*.ti,ab. & 1183145 \\
\hline 26 & (blind*3 or mask³).ti,ab. & 390423 \\
\hline 27 & 'control group'.ti,ab. & 456422 \\
\hline 28 & Placebo\$.ti,ab. & 254276 \\
\hline 29 & or/12-28 & 7298271 \\
\hline 30 & $\begin{array}{l}\text { letter/not (letter/and randomized } \\
\text { controlled trial/) }\end{array}$ & 926792 \\
\hline 31 & Case study/ & 96232 \\
\hline 32 & case report/ & 2206544 \\
\hline 33 & Case report.ti,ab. & 334649 \\
\hline 34 & editorial/ & 553901 \\
\hline 35 & Abstract report/ & 89727 \\
\hline 36 & or/30-35 & 3682083 \\
\hline 37 & 29 not 36 [RCT filter] & 7079237 \\
\hline 38 & 11 and 37 [FASD+Children + RCTs] & 1337 \\
\hline
\end{tabular}

Embase <1974 to 2017 week 10>; date of search: 7 March 2017; search fields: ab, abstract; hw, heading word; kw, author supplied keyword; ti, title; /-, subject heading.

RCTs, randomised controlled trials.

will be used to limit search results to randomised trials. The strategy will be peer reviewed by a second health librarian ${ }^{28}$ and, once finalised, will be adapted for use in Medline (Ovid), Cumulative Index of Nursing and Allied Health Plus with Full text (EBSCO), Cochrane Central Register of Controlled Trials (Cochrane Library-Wiley), PsycINFO (ProQuest) and Proquest Dissertations and Theses (Proquest). Searches will be conducted from inception of database to March 2017.

\section{Other sources}

We will search the WHO's International Clinical Trials Registry Platform and hand-search the most recent 5 years of conference proceedings for the International Conference on Fetal Alcohol Spectrum Disorder (hosted by the University of British Columbia every other year) and the Research Society on Alcoholism (abstracts published in Alcoholism: Clinical and Experimental Research) to identify planned, ongoing or recently completed but unpublished trials of FASD interventions. We will also perform forward searches of all studies included in this review in Web of Science to identify additional citations that might have been missed in the database search. Finally, the reference lists of identified systematic reviews and included trials will be hand-searched for relevant citations. We will 
perform reference management in EndNote (EndNote X6 Thomson-Reuters).

\section{Data collection and analysis \\ Study selection}

A two-step process for study selection will be implemented. First, two reviewers (DS and CM) will independently screen the titles and abstracts (when available) of search results to determine if a study meets inclusion criteria. The reviewers will assess titles/abstracts for studies that meet criteria for: population, intervention and study design. At this stage, authors will not exclude citations on the basis of them not being cited as randomised; however, for a citation to be included, the authors have to describe a comparative study. Each study will be classified as: include, exclude, unclear or duplicate of another citation. The full text of all reports classified as 'include' or 'unclear' by either reviewer will be retrieved for formal review. Next, the two reviewers (DS and CM) will independently assess the full text of each report by using a standardised form that outlines the predetermined inclusion and exclusion criteria. The form will be pilot tested on a sample of studies. After the form is tested, disagreements will be resolved by discussion between the two reviewers or by third-party adjudication (AMAS), as needed.

\section{Data abstraction and management}

Data will be extracted independently by two team members (DS and CM) using a standardised form and entered into a Microsoft Excel database. Data from study reports will be extracted by two team members (DS and CM) independently with disagreements resolved through consensus, and with the assistance of a third party (AMAS) if consensus cannot be achieved.

The following data will be extracted from each study:

1. Author identification;

2. Year of publication;

3. Country of publication;

4. Study methods: study design, study population;

5. Patient characteristics: number of patients enrolled in study, number of patients who did not complete study, type of FASD diagnosis included, mean and median age, sex;

6. Risk of bias criteria (see Assessment of risk of bias section);

7. Intervention and comparator: pharmacological, non-pharmacological, name of specific intervention, type of health professional delivering intervention, type of drugs used, duration of intervention and its comparator;

8. Results reported for the outcomes of interest and time of follow-up of outcomes (ie, immediately after intervention or later in life).

At the end of the review, we will construct a Preferred Reporting Items for Systematic Reviews and Meta-Analyses flow diagram illustrating the number of records and fulltext reports reviewed and either excluded or included.

\section{Assessment of risk of bias}

The internal validity of RCTs will be assessed by using the Cochrane Collaboration Risk of Bias tool ${ }^{29}{ }^{30}$ by two team members (DS and CM). Differences in judgement will be discussed with a third team member (AMAS). This tool consists of six domains (sequence generation, allocation concealment, blinding, incomplete outcome data, selective outcome reporting and 'other' sources of bias) and a categorisation of the overall risk of bias. Each separate domain is judged as 'low risk,' 'unclear risk' or 'high risk.' The overall assessment is based on the responses to individual domains. If one or more individual domains are assessed as having a high risk of bias, the overall judgement will be a high risk of bias. The overall risk of bias will be considered low only if all components are judged as having a low risk of bias. In cases of mixed assessments of low and unclear risk of bias or where all assessments were unclear risk of bias, the overall judgement will be an unclear risk of bias. In addition, information on the source of funding will be collected for each study. Information regarding trial risk of bias will be used to guide sensitivity analyses and explore sources of heterogeneity.

\section{Measures of treatment effect}

The data from included studies will be analysed using RevMan V.5.3.5. A formal meta-analysis will be conducted if the data are sufficiently statistically and clinically homogeneous. If meta-analysis is not appropriate, we will conduct a qualitative synthesis of the evidence using guidance provided by Cochrane (Cochrane Consumers and Communication Review Group: data synthesis and analysis). Pooled continuous data will be expressed as a mean difference or standardised mean difference, where multiple scales are used to measure the same outcome, with $95 \%$ CI. Pooled dichotomous data will be presented as risk ratio or for rare outcomes using the Peto OR. For all studies, we will include data from all reported time periods; separate meta-analyses will be conducted for outcomes measured immediately after intervention, 6 months after and $>12$ months after the intervention. For cluster-randomised trials, we will adjust the reported outcomes to account for the clustering using the interclass correlation coefficient (ICC). If this is not reported, then we will use a range of plausible ICCs and conduct sensitivity analyses to test the roubustness of the reported analyses. For cross-over studies, we will adjust for the lack of independence of the units (similar to the cluster randomised).

\section{Dealing with missing data}

We will attempt to contact authors of included studies in which there are missing data (for example, missing values such as SD, data lost to attrition or statistics or outcomes needed for possible meta-analysis) via email or telephone. Intention-to-treat analyses will be performed whenever possible. 


\section{Subgroup/sensitivity analyses}

We will perform subgroup analyses based on different patient demographic characteristics, differing FASD diagnoses and type of pharmacological intervention if possible. Such analyses will depend on the number of studies included and the availability of appropriate outcomes. We will also attempt to carry out sensitivity analyses that groups studies by different risks of bias.

\section{Assessment of heterogeneity}

We will review both the clinical and statistical heterogeneity of the data using the $\chi^{2}$ Tau-squared and the I-squared statistics. For the I-squared test, we will also review the uncertainty intervals. If significant heterogeneity is suspected, further analysis including subgroup analysis will be conducted.

\section{Assessment of publication bias}

If we are able to meta-analyse the data and more than 10 studies are included in an analysis, publication bias will be assessed by viewing the overlap of the study CIs and by using funnel plot techniques given the known limitations of these methods. ${ }^{31}$

\section{Grading the evidence for each primary outcome}

The strength of evidence for the primary outcomes will be graded by using the approach described by the GRADE working group. ${ }^{32}$ Two reviewers will evaluate the strength of a body of evidence independently, and discrepancies will be resolved through consensus. This approach assesses the evidence based on four domains: risk of bias, inconsistency, indirectness, imprecision, publication bias and other factors (and upgrading). We will classify the strength of evidence as 'high', 'moderate', 'low' or 'very low' and make recommendations for future research needs.

\section{DISCUSSION}

This review will expand on previous reviews in the following ways: (1) provide an updated, comprehensive literature search (from inception of databases to 2016); (2) assess risk of bias of studies using a standardised rating tool; (3) include both pharmacological and non-pharmacological treatments to enhance the relevance to physicians and clinicians administering treatment to children and adolescents with FASD; (4) evaluate the risk of bias from RCTs to provide a review of data generated from the highest quality evidence available, as good quality RCTs are considered the best type of study to compare the effects of a treatment since one can attribute the treatment effects to the interventions being compared and not to confounding factors. ${ }^{33}$

Strengths of this review will include the completeness of the search including searching multiple citation databases, hand-searching relevant conference proceedings, included studies and previous reviews and forward searching and searching clinical trials registry for ongoing trials with no language bias. In addition, authors of ongoing studies will be contacted to obtain unpublished results. Finally, we will use this a priori protocol and follow established methodological guidelines in the conduct and reporting of this review. Further limitations may also arise from the fact that there is high heterogeneity from the methodology used to evaluate FASD treatments.

A limitation of this review is the exclusion of studies using observational study designs, as these types of studies are common when assessing treatments for FASD in community settings. Therefore, this review may be missing effective FASD interventions. Our study team is expanding our programme of research to include a systematic review that summarises studies using observational designs to evaluate FASD interventions.

This review will provide clinicians an updated summary of the evidence generated from RCTs, an appraisal of the risk of bias in existing studies and highlight gaps that can be filled with future studies. This review will provide needed guidance and support for clinicians and researchers by providing a current evidence base for current treatment options for the management of FASD.

\section{Author affiliations}

${ }^{1}$ Manitoba Centre for Health Policy, Department of Community Health Sciences, Max Rady College of Medicine, Rady Faculty of Health Sciences, University of Manitoba, Winnipeg, Manitoba, Canada

${ }^{2}$ Department of Internal Medicine, Max Rady College of Medicine, Rady Faculty of Health Sciences, University of Manitoba, Winnipeg, Manitoba, Canada

${ }^{3}$ Neil John Maclean Health Sciences Library, University of Manitoba, Winnipeg, Manitoba, Canada

${ }^{4}$ Department of Paediatrics and Child Health, Developmental Paediatrics, Max Rady College of Medicine, Rady Faculty of Health Sciences, University of Manitoba, Winnipeg, Manitoba, Canada

${ }^{5}$ Department of Internal Medicine, Section of Critical Care, Max Rady College of Medicine, Rady Faculty of Health Sciences, University of Manitoba, Winnipeg, Manitoba, Canada

${ }^{6}$ Department of Haematology and Medical Oncology, CancerCare Manitoba, Max Rady College of Medicine, Rady Faculty of Health Sciences, University of Manitoba, Winnipeg, Manitoba, Canada

${ }^{7}$ George and Fay Yee Center for Healthcare Innovation, University of Manitoba/ Winnipeg Regional Health Authority, Winnipeg, Manitoba, Canada

Acknowledgements We would like to thank Carol Freiessen for her support in developing the initial search strategy for this review.

Contributors This study was conceived by DS, RZ and AM-S. The manuscript of this protocol was drafted by DS and revised by CM, CJN, MB, AH-D, AC, RZ and AM-S. DS and CN designed the search strategies and CJN will perform the search. DS and CM will independently screen trials for inclusion, extract data and assess the risk of bias of included trials. DS and AM-S will analyse and interpret the data. AM-S will arbitrate any disagreements during the screening, extraction or critical appraisal phases. $\mathrm{MB}, \mathrm{AH}-\mathrm{D}$ and $\mathrm{AC}$ have provided content expertise, and $\mathrm{RZ}$ and AM-S have provided methodological expertise that have guided this paper. All authors approved the final version of this protocol.

Funding This research received no specific grant from any funding agency in the public, commercial or not-for-profit sectors.

Competing interests None declared.

Patient consent Not required.

Provenance and peer review Not commissioned; externally peer reviewed. 
Open Access This is an Open Access article distributed in accordance with the Creative Commons Attribution Non Commercial (CC BY-NC 4.0) license, which permits others to distribute, remix, adapt, build upon this work non-commercially, and license their derivative works on different terms, provided the original work is properly cited and the use is non-commercial. See: http://creativecommons.org/ licenses/by-nc/4.0/

(c) Article author(s) (or their employer(s) unless otherwise stated in the text of the article) 2018. All rights reserved. No commercial use is permitted unless otherwise expressly granted.

\section{REFERENCES}

1. Chudley AE, Conry J, Cook JL, et al. Fetal alcohol spectrum disorder: Canadian guidelines for diagnosis. CMAJ 2005;172:S1-21.

2. Cook JL, Green CR, Lilley CM, et al. Fetal alcohol spectrum disorder: a guideline for diagnosis across the lifespan. CMAJ 2016;188:191-7.

3. Popova S, Lange S, Probst C, et al. Prevalence of alcohol consumption during pregnancy and fetal alcohol spectrum disorders among the general and Aboriginal populations in Canada and the United States. Eur J Med Genet 2017;60:32-48.

4. American Academy of Pediatrics. Committee on Substance Abuse and Committee on Children With Disabilities. Fetal alcohol syndrome and alcohol-related neurodevelopmental disorders. Pediatrics 2000;106:358-61.

5. Popova S, Chambers C. Fetal alcohol spectrum disorders must be recognized globally as a large public health problem. Int $\mathrm{J}$ Alcohol Drug Res 2014;3:1.

6. Bertrand J, Floyd LL, Weber MK. Guidelines for identifying and referring persons with fetal alcohol syndrome. MMWR. Recommendations and reports: morbidity and mortality weekly report.. Recommendations and reports / Centers for Disease Control 2005;54:1-14.

7. Burd L, Cotsonas-Hassler TM, Martsolf JT, et al. Recognition and management of fetal alcohol syndrome. Neurotoxicol Teratol 2003;25:681-8.

8. Peadon E, Elliott EJ. Distinguishing between attention-deficit hyperactivity and fetal alcohol spectrum disorders in children: clinical guidelines. Neuropsychiatr Dis Treat 2010;6:509-15.

9. Streissguth AP. Fetal alcohol syndrome in adolescents and adults. JAMA 1991;265:1961-7.

10. Streissguth AP, Bookstein FL, Barr HM, et al. Risk factors for adverse life outcomes in fetal alcohol syndrome and fetal alcohol effects. $J$ Dev Behav Pediatr 2004;25:228-38.

11. Fryer SL, McGee CL, Matt GE, et al. Evaluation of psychopathological conditions in children with heavy prenatal alcohol exposure. Pediatrics 2007;119:e733-e741.

12. May PA, Gossage JP, Kalberg WO, et al. Prevalence and epidemiologic characteristics of FASD from various research methods with an emphasis on recent in-school studies. Dev Disabil Res Rev 2009;15:176-92.

13. Astley SJ, Bailey D, Talbot C, et al. Fetal alcohol syndrome (FAS) primary prevention through FAS diagnosis: I. Identification of highrisk birth mothers through the diagnosis of their children. Alcohol Alcohol 2000;35:499-508.

14. Paley $\mathrm{B}, \mathrm{O}^{\prime}$ Connor MJ. Intervention for individuals with fetal alcohol spectrum disorders: treatment approaches and case management. Dev Disabil Res Rev 2009;15:258-67.
15. Peadon E, Rhys-Jones B, Bower $\mathrm{C}$, et al. Systematic review of interventions for children with fetal alcohol spectrum disorders. BMC Pediatr 2009;9:35.

16. Premji S, Benzies K, Serrett K, et al. Research-based interventions for children and youth with a fetal alcohol spectrum disorder: revealing the gap. Child Care Health Dev 2007;33:389-97. discussion 98-400.

17. Bertrand J. Interventions for Children with Fetal Alcohol Spectrum Disorders Research Consortium. Interventions for children with fetal alcohol spectrum disorders (FASDs): overview of findings for five innovative research projects. Res Dev Disabil 2009;30:986-1006.

18. Kodituwakku PW. A neurodevelopmental framework for the development of interventions for children with fetal alcohol spectrum disorders. Alcohol 2010;44:717-28.

19. Petrenko CL. Positive behavioral interventions and family support for fetal alcohol spectrum disorders. Curr Dev Disord Rep 2015;2:199-209.

20. Reid N, Dawe S, Shelton D, et al. Systematic review of fetal alcohol spectrum disorder interventions across the life span. Alcohol Clin Exp Res 2015;39:2283-95.

21. Connor PD, Sampson PD, Bookstein FL, et al. Direct and indirect effects of prenatal alcohol damage on executive function. Dev Neuropsychol 2000;18:331-54.

22. Bhatara V, Loudenberg R, Ellis R. Association of attention deficit hyperactivity disorder and gestational alcohol exposure: an exploratory study. J Atten Disord 2006;9:515-22.

23. Doig J, McLennan JD, Gibbard WB. Medication effects on symptoms of attention-deficit/hyperactivity disorder in children with fetal alcohol spectrum disorder. J Child Adolesc Psychopharmacol 2008;18:365-71.

24. O'Malley KD, Koplin B, Dohner VA. Psychostimulant clinical response in fetal alcohol syndrome. Can J Psychiatry 2000;45:90-1.

25. O'Malley KD, Nanson J. Clinical implications of a link between fetal alcohol spectrum disorder and attention-deficit hyperactivity disorder. Can J Psychiatry 2002;47:349-54.

26. Boluyt N, Tjosvold L, Lefebvre C, et al. Usefulness of systematic review search strategies in finding child health systematic reviews in MEDLINE. Arch Pediatr Adolesc Med 2008;162:111-6.

27. Scottish Intercollegiate Guidelines Network. Secondary Scottish Intercollegiate Guidelines Network. Search Filters - Randomized Controlled Trials. QJM 2000;93:359. http://www.sign.ac.uk/ methodology/filters.html - random

28. Peer Review of Electronic Search Strategies. Guideline explanation and elaboration Ottawa. Ottawa: CADTH, 2016.

29. Higgins J, Green S. Cochrane handbook for systematic reviews of interventions version 5.0.1. London, UK: The Cochrane Collaboration, 2008.

30. Higgins JP, Altman DG, Gøtzsche PC, et al. The Cochrane collaboration's tool for assessing risk of bias in randomised trials. BMJ 2011;343:d5928.

31. Ioannidis JP, Trikalinos TA. The appropriateness of asymmetry tests for publication bias in meta-analyses: a large survey. CMAJ 2007;176:1091-6.

32. Guyatt GH, Oxman AD, Vist GE, et al. GRADE: an emerging consensus on rating quality of evidence and strength of recommendations. BMJ 2008;336:924-6.

33. Barton $\mathrm{S}$. Which clinical studies provide the best evidence? The best RCT still trumps the best observational study. BMJ 2000;321:255-6. 